\title{
The Study of Extended Radio Galaxies
}

\author{
M.A.Hovhannisyan \\ Byurakan Astrophysical Observatory, Byurakan, 378433, Armenia
}

The jets of radio galaxies have an essential role in the formation of their extended components. However, in general, the physical characteristics of the jets differ from the characteristics of the extended components (the spectra, polarisation, density and so on).

Detailed observations show that the total jets are in the same shapes as the jets in the core of the galaxies. The study of radio spectra show that the spectra of the core are not all flat. Half of them have steep spectra $(\sim 45 \%)$, but the majority of spectra of the galaxies with radio jets are flat $(\sim 90 \%)$. This proves that an origin of the jets is connected with the younger cores of galaxies, having flat spectra.

However, the jet formation processes are proceeding only in specific physical conditions, for instance, the energies of magnetic field and relativistic electrons must be of the same order. We can draw the final statement that the radio jets are connected with the earlier stage of evolution of galaxies. But in some cases, due to a slow dissipation, the jets can live a relatively long time. Therefore, there are only a small number of galaxies which have radio jets with steep spectra.

\section{References}

Artyukh, V. S., \& Hovhannisyan, M. A. 1988, Pisma AZh, 14, 706

Artyukh, V. S., \& Hovhannisyan, M. A. 1993, AZh, 70, 443

Artyukh, V. S., Hovhannisyan, M. A., \& Tyul'bashev, S. A. 1994, Pisma AZh, 20,178

Artyukh, V. S., Hovhannisyan, M. A., \& Tyul'bashev, S. A. 1994, Pisma AZh, 20,258

Hovhannisyan, M. A. 1995, Astrofizika, 38, 692 